\title{
Respuesta inmune Th1 en la osteoartritis de la articulación temporomandibular
}

\author{
Vernal R*, Velásquez E**, Gamonal J***
}

\section{RESUMEN}

Propósito. La osteoartritis temporomandibular es una enfermedad articular degenerativa, caracterizada por la destrucción del cartílago y hueso articular consecutiva a la respuesta inflamatoria e inmune desarrollada. En el presente trabajo se evalúa la expresión a nivel de ARN mensajero de diversas citoquinas proinflamatorias en los sinoviocitos articulares de individuos afectados por osteoartritis temporomandibular.

Material y métodos. En 12 pacientes afectados de osteoartritis temporomandibular y en 6 sujetos sanos se evaluó la expresión de citoquinas en sinoviocitos de la articulación temporomandibular mediante la técnica de PCR cuantitativa en tiempo real.

Resultados. Significativamente mayores niveles de IL-1 $\beta$, IL-2, IL-12, IL-17, TNF $\alpha$, TNF $\beta$ e IFN $\gamma$ fueron observados en pacientes afectados de osteoartritis temporomandibular en comparación a sujetos sanos. En los sujetos enfermos la citoquina predominante fue IL-12 y en los sanos fue IL-10.

Conclusión. Tomados en conjunto, nuestros datos demuestran una asociación de elevados niveles de citoquinas propias de una respuesta inmune Th1 y la destrucción observada durante la osteoartritis temporomandibular.

Palabras clave: Articulación temporomandibular, citoquinas, respuesta inmune Th1.

\section{SUMMARY}

Background. Temporomandibular osteoarthritis is a degenerative disease that affects the temporomandibular joint and is characterized by the cartilage and bone destruction caused by the inflammatory and immune response. This communication reports on the expression of proinflammatory cytokines in synovial cells of patients with temporomandibular osteoarthritis.

Methods. In twelve temporomandibular osteoarthritis patients and six healthy control subjects, cytokine expression in synovial cells was evaluated by quantitative PCR.

Results. Levels of IL-1 $\beta$, IL-2, IL-12, IL-17, TNF $\alpha$, TNF $\beta$ e IFN $\gamma$ were significantly higher in synovial cells of patients than in controls. In particular, IL-12 was the predominant cytokine in patients and IL-12 in healthy subjects.

Conclusion. Taken together, these data suggest that a Th1 immune response is associated to the destruction observed during the temporomandibular osteoarthritis.

Key words: Temporomandibular joint, cytokines, Th1 immune response.

Fecha de recepción: Diciembre 2006.

Aceptado para publicación: Diciembre 2006.

* Profesor Asistente, Departamento de Odontología Conservadora, Facultad de Odontología, Universidad de Chile.

** Programa de Doctorado, Departamento de Estomatología III, Facultad de Odontología, Universidad Complutense de Madrid, Madrid, España.

*** Profesor Asociado, Depto. de Odontología Conservadora, Facultad de Odontología, Universidad de Chile.

Vernal R, Velásquez E, Gamonal J. Respuesta inmune Th1 en la osteoartritis de la articulación temporomandibular. Av. Odontoestomatol 2007; 23 (2): 75-81. 


\section{INTRODUCCIÓN}

Los trastornos temporomandibulares corresponden a un conjunto de patologías de etiología y sintomatología diversa que afectan a la musculatura masticatoria, las articulaciones temporomandibulares (ATM) y sus estructuras asociadas ${ }^{1,2}$. La osteoartritis (OA) corresponde a un trastorno degenerativo de las ATM caracterizado por una degradación del cartílago articular y una destrucción ósea reaccional $^{1,2}$. Variables grados de inflamación han sido descritos en la literatura científica, en donde se ha propuesto que varios mediadores inflamatorios, tales como interleuquina (IL) -1 , IL-6, factor de necrosis tumoral-alfa (TNF $\alpha$ ) y prostaglandina $\mathrm{E}_{2}$ $\left(\mathrm{PGE}_{2}\right)$, servirían como marcadores de activa degeneración de la ATM durante la $\mathrm{OA}^{3,4}$. A pesar de los recientes avances en el conocimiento de las bases celulares y moleculares de la patofisiología de la OA muchos aspectos de su etiopatogenia quedan aún por resolver. El objetivo de este trabajo es determinar el tipo de respuesta inflamatoria predominante en las ATM de individuos afectados de OA, a través de la evaluación de los niveles de IL$1 \beta$, IL-2, IL-4, IL-5, IL-6, IL-10, IL-12, IL-17, TNF $\alpha$, TNF $\beta$ e IFN $\gamma$ presentes, mediante la técnica de Reacción en Cadena de la Polimerasa Cuantitativa (qPCR).

\section{MATERIAL Y MÉTODOS}

\section{Selección de los pacientes}

Fueron estudiados 12 pacientes ( 4 hombres y 8 mujeres con edad promedio de $41,4 \pm 2,4$ años) con el diagnóstico clínico de OA temporomandibular. Los criterios clínicos diagnósticos fueron: Impedimento de la movilidad articular, dolor articular y cambios degenerativos en las superficies óseas articulares evaluada mediante tomografía. Los pacientes fueron evaluados y los datos clínicos fueron registrados por 2 evaluadores independientes y la toma de muestra se procedió a realizar habiendo interrumpido el tratamiento farmacológico indicado en al menos 2 semanas previas. Como grupo control fueron evaluados 6 individuos sanos (2 hombres y 4 mujeres con edad promedio de $39,0 \pm 2,2)$.

\section{Colección de la muestra}

Las muestras de fluido sinovial fueron obtenidas mediante anestesia local extracapsular de la ATM. $2 \mathrm{~mL}$ de suero fisiológico fue inyectado en el compartimiento superior de la articulación y 1 minuto después $2 \mathrm{~mL}$ de una mezcla de solución salina y fluido sinovial fue aspirada y centrifugada para la obtención de células sinoviales, las que fueron inmediatamente sumergidas en $5 \mathrm{~mL}$ medio de cultivo estéril RPMI 1640 suplementado con $50 \mathrm{UI} / \mathrm{mL}$ de penicilina, $50 \mu \mathrm{g} / \mathrm{mL}$ de estreptomicina, $200 \mathrm{mM}$ de L-glutamina (Sigma Chemical Co, St Louis, MO, USA) y $10 \%$ de suero bovino fetal (Gibco Invitrogen, Grand Island, NY, USA), y mantenidas a $4^{\circ} \mathrm{C}$ para posterior extracción de ARN total.

\section{Extracción de ARN total}

Previo lavado 2 veces en PBS, las células fueron resuspendidas en $400 \mu \mathrm{L}$ de amortiguador de lisis NP40 suplementado con VRC $10 \mathrm{mM}$ y sometidas a agitación constantemente durante 10 segundos hasta lograr completa lisis celular. Posterior a centrifugación a $18.000 \mathrm{xg}, 350 \mu \mathrm{L}$ de sobrenadante fue resuspendido en $20 \mu \mathrm{L}$ de SDS, $12 \mu \mathrm{L}$ de EDTA 0,5 M y $10 \mu \mathrm{L}$ de proteinasa $\mathrm{K} 10 \mathrm{mg} / \mathrm{mL}$, para ser incubadas durante 30 minutos a $37^{\circ} \mathrm{C}$. $400 \mu \mathrm{L}$ de fase acuosa se obtuvo luego de agitación vigorosa en $400 \mu \mathrm{L}$ de cloropán durante un minuto, a partir de la cual se procedió a la precipitación del ARN en $40 \mu \mathrm{L}$ de acetato de sodio 3 M, $1 \mu \mathrm{L}$ de glicógeno y $1 \mathrm{~mL}$ de etanol $100 \%$ durante al menos 1 hora a $-20^{\circ} \mathrm{C}$. El ARN total se precipitó en $20 \mu \mathrm{L}$ de agua libre de ARNasas.

\section{Construcción de la primera cadena de cDNA}

La primera cadena de cADN se construyó utilizando el kit Transcriptor First Strand cDNA Synthesis, de acuerdo a las instrucciones del fabricante (Roche Applied Science, Mannhein, Germany). Brevemente, $1 \mu \mathrm{g}$ de ARN total fue retrotranscrito en termociclador (MWG AG Biotech, Germany) en $20 \mu \mathrm{L}$ de volumen final de primeros hexaméricos aleatorios $60 \mu \mathrm{M}$, inhibidor de ARNasas 20 U, mezcla de deoxinucleótidos $1 \mathrm{mM}$ y enzima transcriptasa reversa $10 U$ en agua libre de ARNasas, en las siguientes condiciones: 
Incubación inicial a $25^{\circ} \mathrm{C}$ durante 10 minutos y luego 2 incubaciones a $50^{\circ} \mathrm{C}$ durante 1 hora y $85^{\circ} \mathrm{C}$ durante 5 minutos. Las muestras fueron almacenadas a $-20^{\circ} \mathrm{C}$ hasta posterior amplificación mediante qPCR.

\section{PCR cuantitativo}

Para evaluar a expresión de IL-1 $\beta$, IL-2, IL-4, IL-5, IL6, IL-10, IL-12, IL-17, TNF $\alpha$, TNF $\beta$ e IFN $\gamma$ se procedió a realizar amplificación en termociclador en tiempo real ABI PRISMTM 7700 Sequence Detector System (Applied Biosystems, CA, USA) utilizando el kit FastStart TaqMan Probe Master (Roche Applied Science). Brevemente, $1 \mu \mathrm{g}$ de cADN fue amplificado en $20 \mu \mathrm{L}$ de volumen final con FastStart TaqMan Master 1x, sonda de hidrólisis $250 \mathrm{nM}$, primer específicos (Tabla 1) 900 nM cada uno, en agua libre de ARNasas en las siguientes condiciones: 1 ciclo de $95^{\circ} \mathrm{C}$ durante 10 minutos y 40 ciclos de $95^{\circ} \mathrm{C}$ durante 15 segundos y $60^{\circ} \mathrm{C}$ durante 1 minuto.

\section{Análisis de los datos}

Los datos fueron expresados como Ct, $\Delta \mathrm{Ct}$ y cuantificación relativa $R Q$ y analizados con el software esta- dístico SPSS 13.0 (Lead Technologies Inc., Charlote, NC, USA). El test Shapiro-Wilk fue utilizado para determinar distribución normal de lo datos. La prueba estadística $t$ de Student no pareada de 2 colas fue utilizada para analizar cada citoquina entre el grupos control y experimental. La prueba ANOVA no pareada de 2 colas y la prueba de Tukey se utilizaron para comparar los niveles de citoquinas dentro del grupo control y del experimental. Un $\mathrm{p}<0,05$ fue considerado como diferencia estadísticamente significativa.

\section{RESULTADOS}

Los valores $\mathrm{Ct}$ de cada citoquina y los valores $\Delta \mathrm{Ct}$ de cada citoquina ajustada al gen housekeeping $18 \mathrm{~S}$ se resumen en la tabla 2 . El Ct de $18 \mathrm{~S}$ fue similar entre grupo experimental y control $(22,288 \pm 1,94$ vs. $22,349 \pm 1,34)$. Los valores $\mathrm{Ct}$ y $\Delta$ Ct de IL-1 $\beta$, IL-2, IL-12, IL-17, TNF $\alpha$, TNF $\beta$ e IFN $\gamma$ fueron significativamente mayores en pacientes con OA temporomandibular en comparación a sujetos sanos. El Ct, pero no el $\Delta \mathrm{Ct}$, de IL-5 fue significativamente mayor en pacientes con OA versus controles sanos. Al analizar la cuantificación relativa $R Q$ de cada citoquina (Tabla 3) se observó que IL-12 fue la que se sobre-expresó con mayor intensidad

\begin{tabular}{|c|c|c|c|}
\hline \multicolumn{4}{|c|}{$\begin{array}{l}\text { TABLA 1.- SONDAS Y FORWARD Y REVERSE PRIMERS UTILIZADOS LA AMPLIFICACIÓN } \\
\text { MEDIANTE qPCR DE LAS CITOQUINAS ESTUDIADAS Y DEL GEN CONTROL INTERNO 18S }\end{array}$} \\
\hline Citoquina & Forward Primer & Reverse Primer & Sonda* \\
\hline IL-1 $\beta$ & ctgtcctgcgtgttgaaaga & ttgggtaatttttgggatctaca & 78 \\
\hline IL-2 & aagttttacatgcccaagaagg & aagtgaaagttttgctttgagc & 65 \\
\hline IL-4 & caccgagttgaccgtaacag & gccctgcagaaggtttcc & 16 \\
\hline IL-5 & ctctgaggattcctgttcctgt & cagtaccccettgcacagtt & 47 \\
\hline IL-6 & gcccagctatgaactccttct & gaaggcagcaggcaacac & 45 \\
\hline IL-10 & tgggggagaacctgaagac & ccttgctcttgttttcacagg & 30 \\
\hline IL-12 p35 & cactcccaaaacctgctgag & tctcttcagaagtgcaagggta & 50 \\
\hline IL-12 p40 & ccctgacattctgcgttca & aggtcttgtccgtgaagactcta & 37 \\
\hline IL-17 & tgggaagacctcattggtgt & ggatttcgtgggattgtgat & 8 \\
\hline TNF $\alpha$ & cagcctcttctccttcctgat & gccagagggctgattagaga & 29 \\
\hline TNF $\beta$ & ctaccgcccagcagtgtc & gtggtgtcatggggaga & 13 \\
\hline $\mathrm{IFN \gamma}$ & ggcattttgaagaattggaaag & tttggatgctctggtcatctt & 21 \\
\hline $18 S$ & ctcaacacgggaaacctcac & cgctccaccaactaagaacg & 77 \\
\hline
\end{tabular}

IL: Interleukina; TNF: Factor de necrosis tumoral; 18S: gen housekeeping de la sub-unidad 18S ribosomal.

* Sonda de hidrólisis FAM-conjugada (Roche Applied Science, Mannhein, Germany). 
AVANCES EN ODONTOESTOMATOLOGÍA

Vol. 23 - Núm. 2 - 2007

TABLA 2.- Ct Y $\Delta$ Ct DE IL-1 $\beta$, IL-2, IL-4, IL-5, IL-6, IL-10, IL-12, IL-17, TNF $\alpha$, TNF $\beta$ E IFN $\gamma$ EVALUADAS MEDIANTE QPCR EN PACIENTES CON OSTEOARTRITIS TEMPOROMANDIBULAR Y CONTROLES SANOS

\begin{tabular}{|c|c|c|c|c|}
\hline & \multicolumn{2}{|c|}{$\begin{array}{l}\text { Osteoartritis temporomandibular } \\
\qquad \mathrm{N}=12\end{array}$} & \multicolumn{2}{|c|}{$\begin{array}{l}\text { Controles sanos } \\
\qquad \mathrm{N}=6\end{array}$} \\
\hline & $\mathrm{Ct}$ & $\Delta \mathrm{Ct}$ & $\mathrm{Ct}$ & $\Delta \mathrm{C}$ \\
\hline IL-1 $\beta$ & $33,198 \pm 1,04 \S$ & $10,918 \pm 2,13 \dagger$ & $36,250 \pm 1,02 \S$ & $13,901 \pm 2,21$ \\
\hline IL-2 & $32,985 \pm 1,20 \S$ & $10,705 \pm 2,07 \dagger$ & $35,750 \pm 1,39 \S$ & $13,401 \pm 2,34$ \\
\hline IL-4 & $36,424 \pm 1,48$ & $14,144 \pm 1,38$ & $36,625 \pm 1,27$ & $14,276 \pm 2,22$ \\
\hline IL-5 & $35,895 \pm 1,45 \dagger$ & $13,615 \pm 1,26$ & $37,218 \pm 0,68 \dagger$ & $14,869 \pm 1,61$ \\
\hline IL-6 & $37,748 \pm 2,04$ & $15,468 \pm 2,93$ & $38,270 \pm 1,35$ & $15,921 \pm 1,33$ \\
\hline IL-10 & $37,247 \pm 1,62$ & $14,967 \pm 2,61$ & $36,465 \pm 0,96$ & $14,116 \pm 1,71$ \\
\hline IL-12 p35 & $23,946 \pm 1,52 \S$ & $1,666 \pm 1,42 \S$ & $28,930 \pm 2,96 \S$ & $6,581 \pm 2,96$ \\
\hline IL-12 p40 & $25,035 \pm 2,34 \S$ & $2,755 \pm 1,55 \S$ & $29,960 \pm 1,73 \S$ & $7,611 \pm 1,48$ \\
\hline IL-17 & $29,822 \pm 1,42 \ddagger$ & $7,542 \pm 2,31 \ddagger$ & $33,769 \pm 2,35 \neq$ & $11,420 \pm 2,49$ \\
\hline TNF $\alpha$ & $32,477 \pm 1,51 \S$ & $10,197 \pm 2,56 \dagger$ & $35,127 \pm 0,95 \S$ & $12,778 \pm 1,96$ \\
\hline TNF $\beta$ & $31,977 \pm 1,65 \ddagger$ & $9,697 \pm 2,25 \dagger$ & $34,730 \pm 1,03 \ddagger$ & $12,381 \pm 1,67+$ \\
\hline IFNy & $23,893 \pm 1,94 \S$ & $1,613 \pm 2,49 \ddagger$ & $27,977 \pm 1,53 \S$ & $5,628 \pm 1,19$ \\
\hline $18 S$ & $22,288 \pm 1,94$ & & $22,349 \pm 1,34$ & \\
\hline
\end{tabular}

Ct: Ciclo umbral; $\quad$ DCt=Ct citoquina - Ct $18 S$

$\S p<0,001$

$+p<0,05$

TABLA 3.- CUANTIFICACIÓN RELATIVA DE IL-1ß, IL-2, IL-4, IL-5, IL-6, IL-10, IL-12, IL-17, TNF $\alpha$, TNF $\beta$ E IFN $\gamma$ EVALUADAS MEDIANTE qPCR EN PACIENTES CON OSTEOARTRITIS TEMPOROMANDIBULAR Y CONTROLES SANOS

\begin{tabular}{|c|c|c|c|c|}
\hline & \multicolumn{2}{|c|}{$\begin{array}{l}\text { Osteoartritis temporomandibular } \\
\qquad \mathrm{N}=12\end{array}$} & \multicolumn{2}{|c|}{$\begin{array}{l}\text { Controles sanos } \\
\qquad \mathrm{N}=6\end{array}$} \\
\hline & $\mathbf{R Q}$ & Rango de error & $\mathbf{R Q}$ & Rango de error \\
\hline IL-1 $\beta$ & 7,9 & $1,8-34,6$ & 1,0 & $0,2-4,6$ \\
\hline IL-2 & 6,5 & $2,5-27,2$ & 1,0 & $0,2-5,0$ \\
\hline IL-4 & 1,1 & $0,4-2,8$ & 1,0 & $0,2-4,7$ \\
\hline IL-5 & 2,4 & $1,0-5,7$ & 1,0 & $0,3-3,1$ \\
\hline IL-6 & 1,4 & $0,2-10,5$ & 1,0 & $0,4-2,5$ \\
\hline IL-10 & 0,6 & $0,1-3,4$ & 1,0 & $0,3-3,3$ \\
\hline IL-12 p35 & 30,2 & $11,2-81,0$ & 1,0 & $0,1-7,8$ \\
\hline IL-12 p40 & 29,0 & $9,9-85,0$ & 1,0 & $0,4-2,8$ \\
\hline IL-17 & 14,7 & $3,0-72,9$ & 1,0 & $0,2-5,6$ \\
\hline TNF $\alpha$ & 6,0 & $1,0-35,4$ & 1,0 & $0,3-3,9$ \\
\hline TNF $\beta$ & 6,4 & $1,3-30,6$ & 1,0 & $0,3-3,2$ \\
\hline IFN $\gamma$ & 16,2 & $2,0-90,8$ & 1,0 & $0,4-2,3$ \\
\hline
\end{tabular}

RQ: Cuantificación relativa. Niveles de la citoquina en estudio en el grupo experimental y ajustado al gen housekeeping $18 S$ cuando esta relación es 1 en el grupo control sano. 
en sujetos con OA temporomandibular (30,2 y 29,0 para IL-12 p35 e IL-12 p40, respectivamente), IL$1 \beta$, IL-2, IL-17, TNF $\alpha$, TNF $\beta$ e IFN $\gamma$ se sobreexpresaron en cantidades moderadas e IL-10 se observó expresado con mayor intensidad en individuos controles sanos.

\section{DISCUSIÓN}

Durante la OA temporomandibular se desarrolla un complejo proceso inflamatorio que involucra la síntesis de una serie de citoquinas por parte de los sinoviocitos y células infiltrantes inflamatorias, principalmente del tipo linfocitario $\mathrm{T}^{1,2}$. La cascada de mediadores inflamatorios secretados lleva a la sobreexpresión de citoquinas involucradas en la activación de fibroblastos que secretan metaloproteinasas y de precursores celulares de osteoclastos, mediante citoquinas osteoactivas, que llevan a diferenciación y activación de osteoclastos, que en definitiva causan la destrucción de la matriz orgánica y mineral del cartílago y hueso articular, característicos de la patología ${ }^{1,2}$.

Diferentes mediadores inflamatorios han sido propuestos como potenciales marcadores de la degeneración articular durante la $\mathrm{OA}^{3,4}$. Estudios recientes han evaluado la asociación de citoquinas proinflamatorias y trastornos temporomandibulares diversos, en donde, mediante ELISA, se ha establecido que IL-1, IL-6, TNF $\alpha$, y $\mathrm{PGE}_{2}$ se encuentran asociados al daño articular ${ }^{4,5}$.

Nuestro estudio evaluó los niveles de distintas citoquinas en su asociación al diagnóstico clínico de AO temporomandibular. Mediante qPCR se observó significativamente mayores niveles de IL-1 $\beta$, IL-2, IL12, IL-17, TNF $\alpha$, TNF $\beta$ e IFN $\gamma$ en sujetos enfermos.

Los linfocitos $\mathrm{T}$ ayudadores (Th) del tipo CD4 ${ }^{+}$pueden funcionalmente se divididos en al menos 2 subtipos, Th1 y Th2, de acuerdo al patrón de citoquinas que ellos expresan al ser activados ${ }^{6}$. Una respuesta Th1 se caracteriza por un predominio en la síntesis de citoquinas IL-1 $\beta$, IL-2, IL-12, TNF $\beta$ e IFN $\gamma$ y se relaciona con una respuesta de tipo celular, en donde neutrófilos y macrófagos cumplen un rol esencial en el desarrollo de la patología?. Por otro lado, una respuesta Th2 se caracteriza por la expresión de citoquinas tipo IL-4, IL-5, IL-6 e IL-13, y se relaciona con una respuesta del tipo humoral, en donde predomina la síntesis de inmunoglobulinas reactivas a los antígenos desencadenantes de la respuesta inmune?

Entre los factores determinantes del tipo de respuesta Th desarrollado se puede enumerar: El tipo de antígeno desencadenante de la respuesta, como este antígeno es presentado por las células presentadoras de antígeno (APCs) a los linfocitos T efectores, el tipo se señales co-estimulatorias expresadas por las APCs, y, principalmente, el ambiente de citoquinas existente $^{8}$. A este respecto, se ha establecido que IL12 promueve la diferenciación a un fenotipo Th1, mientas IL-4 promueve la diferenciación Th2 ${ }^{8}$.

En la actualidad, respuestas distintas al tipo Th1 o Th2 han sido propuestas, tales como, Th17 dependiente de un predominio de IL-17, y respuesta Th3, asociada a un predominio de IL- $10^{9,10}$, profundizado el conocimiento en el tipo de citoquina sintetizado durante el proceso inflamatorio y enfatizando la importancia del tipo de respuesta en el resultado final de la enfermedad ${ }^{11}$.

IL-10 y TGF $\beta$ (factor de crecimiento transformantebeta) se han descrito como citoquinas inmunosupresivas y se han propuesto como mediadores esenciales en la diferenciación y actividad de linfocitos $T$ reguladores tipo $1(\operatorname{Tr} 1)$ y $\operatorname{Th} 3^{12}$. Las propiedades inmunosupresivas de las células Tr1 y T3 pueden probablemente ser explicadas por la propiedad de las citoquinas en inhibir la función de las APCs. Células dendríticas tratadas in vitro con IL-10 disminuyen la síntesis de citoquinas proinflamatorias, incluyendo IL-1 $\beta$, IL-6, TNF $\alpha$ e IL-12, inhibiendo la inducción de células Th1 específicas al antígeno y regulando la respuesta de las células $T$ efectoras ${ }^{13}$.

En forma similar a reportes previos, nuestros datos muestran que mayores niveles de citoquinas se encuentran asociados a desórdenes de la ATM en comparación a controles sanos ${ }^{5,14,15}$. A partir de los datos obtenidos de nuestro estudio se puede concluir que una respuesta del tipo Th1 se establece en las ATM de individuos afectados de $\mathrm{OA}$, en donde un predominio de IL-12 es característico de la lesión. Por otro lado, 
sujetos sanos muestran un predominio de IL-10, pudiéndose conjeturar una potencial respuesta reguladora, en donde linfocitos Th3 cumplirían un rol fundamental durante la salud articular. Una respuesta Th1, activaría mecanismos asociados a la síntesis de metaloproteinasas y de RANKL por parte de los fibroblastos articulares, este último asociado a la estimulación de la diferenciación y activación de monocitos a osteoclastos maduros, desencadenándose la destrucción del cartílago y hueso articular durante la OA de las ATM $^{16,17}$.

En el futuro, la evaluación de las citoquinas proinflamatorias como marcadores de la destrucción articular podrían ser útiles en el diagnóstico y monitoreo de los desórdenes de la ATM. Nuevos estudios son necesarios para dilucidar la distribución temporoespacial de las citoquinas, la relación sinérgica y antagonista de ellas, el rol de su relación a sus receptores específicos y los mecanismos regulatorios involucrados en la aparición y desarrollo de la enfermedad.

\section{BIBLIOGRAFÍA}

1. Velasco E, Cruz D, Velasco C, Monsalve L, Bullón P. Los trastornos temporomandibulares en la práctica odontológica. I. Clasificación y Etiopatogenia. Av Odontoloestomatol. 2002;18:177-89.

2. Velasco E, Cruz D, Velasco C, Monsalve L, Paz J. Los Trastornos temporomandibulares en la práctica odontológica. II. El Diagnóstico. Av Odontoloestomatol. 2002;18: 211-9.

3. Sandler NA, Buckley MJ, Cillo JE, Braun TW. Correlation of inflammatory cytokines with arthroscopic findings in patients with temporomandibular joint internal derangements. J Oral Maxillofac Surg. 1998;56:534-43.

4. Shafer DM, Assael L, White LB, Rossomando EF. Tumor necrosis factor-alpha as a biochemical marker of pain and outcome in temporomandibular joints with internal derangements. J Oral Maxillofac Surg. 1994;52:786-91.

5. Kaneyama K, Segami N, Sun W, Sato J, Fujimura K. Analysis of tumor necrosis factor-a, interleukin-6, interleukin-1b, soluble tumor necrosis factor receptors I and II, interleukin- 6 soluble receptor, interleukin-1 soluble receptor type II, interleukin-1 receptor antagonist, and protein in the synovial fluid of patients with temporomandibular joint disorders. Oral Surg Oral Med Oral Pathol Oral Radiol Endod. 2005;99:27684.

6. Abbas AK, Murphy KM, Sher A. Functional diversity of helper $\mathrm{T}$ lymphocytes. Nature. 1996;383:787-93.

7. Romagnani S. Biology of human $\mathrm{TH} 1$ and $\mathrm{TH} 2$ cells. J Clin Immunol. 1995;15:121-9.

8. O'Garra A, Arai N. The molecular basis of T helper 1 and T helper 2 cell differentiation. Trends Cell Biol. 2000;10:542-50.

9. Weaver CT, Harrington LE, Mangan PR, Gavrieli M, Murphy KM. Th17: an effector CD4 T cell lineage with regulatory $\mathrm{T}$ cell ties. Immunity. 2006;24:677-88.

10. Azuma M. Fundamental mechanisms of host immune response to infection. J Periodont Res. 2006;41:361-73.

11. Cousins DJ, Lee TH, Staynov DZ. Cytokine coexpression during human Th1/Th2 cell differentiation: Direct evidence for coordinated expression of Th2 cytokines. J Immunol. 2002; 169:2498-506.

12. Groux H, O'Garra A, Bigler M et al. A CD4+ T-cell subset inhibits antigen-specific $\mathrm{T}$-cell responses and prevents colitis. Nature. 1997;389:737-42.

13. Jonuleit H, Schmitt E, Steinbrink K, Enk AH. Dendritic cells as a tool to induce anergic and regulatory T cells. Trends Immunol. 2001;22:394400.

14. Takahashi T, Kondoh T, Fukuda $M$ et al. Proinflammatory cytokines detectable in synovial fluids from patients with temporomandibular disorders. Oral Surg Oral Med Oral Pathol Oral Radiol Endod. 1998;85:135-41. 
15. Kaneyama K, Segami N, Nishimura M, Suzuki T, Sato J. Importance of proinflammatory cytokines in synovial fluid from 121 joints with temporomandibular disorders. Br J Oral Maxillofac Surg. 2002;40:418-23.

16. Carda C, Silvestrini G, Gomez de Ferraris ME, Peydró A, Bonucci E. Osteoprotegerin (OPG) and RANKL expression and distribution in developing human craniomandibular joint. Tissue Cell. 2005;37:247-55.

17. Wakita T, Mogi M, Kurita K, Kuzushima M, Togari A. Increase in RANKL:OPG ratio in synovia of patients with temporomandibular joint disorder. J Dent Res 2006;85: 627-32.

\section{CORRESPONDENCIA}

Rolando Vernal Astudillo Laboratorio de Biología Periodontal

Facultad de Odontología - Universidad de Chile Olivos 943 Independencia

Santiago - Chile

Teléfono: +56-2-9781833. Fax: +56-2-9781813

E-mail: rvernal@uchile.cl 